\title{
Intraoperative Schnellschnittuntersuchungen parapylorischer Lymphknoten bei der pyloruserhalten- den Pankreaskopfresektion: Gibt es eine klinische Relevanz?
}

\author{
Hartwig Riediger Antje Schulz Ulrich Adam Colin M. Krüger \\ Klinik für Chirurgie - Viszeral- und Gefäßchirurgie, Vivantes-Humboldt-Klinikum, Berlin, Deutschland
}

Schlüisselwörter

Pankreaskarzinom · Parapylorische Lymphknoten .

Pankreaskopfresektion . PPPD

\section{Zusammenfassung}

Hintergrund: Die pyloruserhaltende Pankreaskopfresektion (PPPD) ist als onkologisches Standardverfahren etabliert. Lokal fortgeschrittene Tumoren können eine erweiterte Resektion erforderlich machen. Ebenso soll früheren Arbeiten zufolge bei Tumornachweis in den parapylorischen Lymphknoten (PLK) eine distale Magenresektion im Sinne einer klassischen Whipple-Operation indiziert sein. Entsprechend diesen Empfehlungen haben wir intraoperative Schnellschnittuntersuchungen der PLK in unseren Routineablauf integriert. Im Rahmen dieser Studie haben wir die klinische Relevanz dieses Vorgehens hinterfragt. Methoden: Bei 105 onkologischen Patienten im Zeitraum von 2006-2012 bestand die Indikation zur PPPD. In allen Fällen erfolgte eine intraoperative Schnellschnittuntersuchung der PLK. Die Patienten wurden bezüglich Primärtumor, Anzahl der untersuchten Lymphknoten (LK) (gesamt und parapylorisch) sowie Auswirkungen auf das operative Konzept untersucht. Es handelt sich um eine retrospektive Studie, die auf prospektiv erhobenen Daten unserer Pankreasdatenbank basiert. Ergebnisse: Die Primärtumoren waren 72 Pankreaskopfkarzinome und 33 extrapankreatische Karzinome (Gallengangskarzinom, Ampullenkarzinom, Duodenalkarzinom). 73 Patienten waren nodalpositiv. Insgesamt wurden 2391 LK untersucht, von denen 325 parapylorisch lokalisiert waren. Die intraoperative Schnellschnittuntersuchung erbrachte lediglich bei 4 Patienten mit Pankreaskopfkarzinom jeweils einen positiven PLK; daraufhin erfolgte eine distale Magenresektion. In keinem der distalen Magenresektate waren Tumorresiduen nachweisbar. Lokale chirurgisch-technische Probleme im Sinne von Durchblutungsstörungen des Magens ergaben sich durch die regionale Lymphadenektomie nicht. PLK waren nur beim Pankreaskarzinom positiv. In der Subgruppe der nodalpositiven Patienten mit Pankreaskopfkarzinom hatten $8 \%$ der Patienten einen positiven PLK. Schlussfolgerung: Die regionale parapylorische Lymphadenektomie ist beim Pankreaskarzinom in einigen (5\%) Fällen onkologisch sinnvoll. Der Nutzen einer intraoperativen Schnellschnittuntersuchung mit nachfolgender Konsequenz für eine etwaige distale Magenresektion ist anhand unserer Daten nicht belegbar.
Keywords

Pancreatic carcinoma - Peripyloric lymph nodes . Pancreatoduodenectomy · PPPD

\section{Summary}

Fresh-Frozen Section of Peripyloric Lymph Nodes in Pylorus-Preserving Pancreatoduodenectomy: Is There any Clinical Benefit?

Background: Pylorus-preserving pancreatoduodenectomy (PPPD) has become one of the most preferred procedures in pancreatic surgery for oncologic indications. Locally advanced tumors may indicate extended resections. Similarly, according to some authors, a tumor infiltration of peripyloric lymph nodes (PLN) is supposed to indicate a distal gastric resection (Whipple procedure). We adopted these pieces of advice and performed a freshfrozen section of PLN in the context of our routine work-up. With this study, we evaluate our clinical findings. Methods: From 2006 to 2012, 105 oncologically indicated PPPD were planned. In every case, fresh-frozen section of PLN was performed during the procedure. For the purpose of this study, a work-up of perioperative outcome and histopathological findings was performed. Data were retrieved from our pancreatic database. Results: Primary tumors consisted of 72 pancreatic head carcinoma and 33 extrapancreatic carcinoma (distal bile duct cancer, ampullary cancer, duodenal cancer). 73 patients had node-positive tumors. A total of 2,391 lymph nodes were examined. 325 lymph nodes were peripyloric. In only 4 patients with pancreatic head cancer, one positive lymph node each was found and lower gastric resection was performed. No tumor was found in the resected distal stomach. PLN resection did not impair the gastric blood flow. Only in pancreatic cancer, the PLN were affected by the tumor. In nodepositive pancreatic cancer, $8 \%$ of all patients showed a proof of tumor infiltration in PLN. Conclusion: The resection of PLN may have oncologic advantages in some $(5 \%)$ cases of pancreatic head cancer. However, a benefit of fresh-frozen section and, additionally, a lower gastric resection cannot be concluded from our data.

\section{KARGER \\ Fax +497614520714 \\ Information@Karger.com}

www.karger.com (c) 2014 S. Karger GmbH, Freibur

662-6664/14/0301-0061\$39.50/0

Accessible online at:

www.karger.com/vim
Dr. Hartwig Riediger

Klinik für Chirurgie - Visceral- und Gefäßchirurgie

Vivantes-Humboldt-Klinikum

Am Nordgraben 3, 13503 Berlin, Deutschland

Hartwig.Riediger@vivantes.de 


\section{Einleitung}

Die kurative R0-Resektion stellt nach wie vor die einzige Option auf Heilung beim Pankreaskarzinom bzw. periampullären Karzinom dar. In den meisten Kliniken hat sich hierbei die pyloruserhaltende Pankreaskopfresektion (pylorus-preserving pancreatoduodenectomy (PPPD)) etabliert. Sie wurde 1971 von Traverso und Longmire beschrieben und hat sich seither in vielen Studien im Vergleich zur klassischen KauschWhipple-Operation mit distaler Magenresektion als onkologisch ebenbürtiges Verfahren erwiesen [1-3]. Das Resektionsausmaß der PPPD ist heute relativ einheitlich definiert. Frühere Ansätze einer erweiterten Lymphadenektomie insbesondere im Bereich der Arteria mesenterica superior (AMS) wurden aufgrund einer deutlich erhöhten Morbidität wieder verlassen. Die Standardlymphadenektomie beschänkt sich auf die rechte Seite der AMS und soll eine Mindestzahl von 12 Lymphknoten (LK) erreichen. Die parapylorischen Lymphknoten (PLK) gehören nicht regelhaft zur Standardlymphadenektomie. Sie entsprechen der Lymphknotenstation Nr. 6 der Japanese Gastric Cancer Association.

Gleichwohl sind die perigastrischen Lymphknotenstationen auch in den letzten Jahren immer wieder das Thema kleinerer Serien gewesen. Ein Tumorbefall der PLK wurde in bis zu 1020\% aller Patienten mit Pankreaskarzinom nachgewiesen, wohingegen andere Primärtumoren der periampullären Region deutlich seltener betroffen waren [4, 5]. Nach Meinung mancher Autoren ergab sich die Empfehlung, beim Tumorbefall der PLK eine distale Magenresektion durchzuführen [6]. Im eigenen Krankengut haben wir die beschriebene Vorgehensweise adaptiert und intraoperative Schnellschnittuntersuchungen der PLK durchgeführt. Bei Tumorbefall erfolgte - abweichend von der normalerweise geplanten PPPD - eine klassische Pankreaskopfresektion nach Whipple.

Im Rahmen dieser retrospektiven Auswertung beschreiben wir die Ergebnisse unseres Konzepts und hinterfragen die klinische Relevanz des Vorgehens. Es handelt sich um eine konsekutive Patientengruppe, die mit Methoden der histopathologischen Routinediagnostik untersucht wurde.

\section{Patienten und Methode}

Im Zeitraum von November 2006 bis Mai 2012 wurde bei 105 Patienten (50 weiblich, 55 männlich) aufgrund der präoperativen Befunde und der Anamnese ein Pankreaskopftumor oder periampullärer Tumor vermutet. Das mediane Patientenalter betrug 70 Jahre (39-87 Jahre).

Bei entsprechender Klinik und Symptomatik ist die Indikation zur onkologischen Pankreaskopfresektion bei Vorliegen folgender Bildbefunde gegeben: i) bildgebender Nachweis einer Tumorformation, ii) kein Nachweis von Fernmetastasen, iii) keine Infiltration der Arterien, iv) kein Tumorverschluss der Pfortaderachse. Eine Computertomographie erfolgte in allen Fällen. Nach individuellem Vorgehen wurde diese durch eine Endosonographie bzw. Magnetresonanztomographie ergänzt. Eine präoperative Biopsie wird an unserer Klinik grundsätzlich nicht durchgeführt. Die Bestimmung von CA 19-9 als Tumormarker erfolgt routinemäßig.
Bei allen Patienten war auf Grundlage der präoperativen Befunde die Indikation zur PPPD gegeben, die in einheitlicher Weise durch zwei verschiedene Operateure durchgeführt wurde. Intraoperative Schnellschnittuntersuchungen erfolgten an drei Regionen: i) distaler Ductus hepaticus, ii) Absetzungsrand des Pankreas über der Pfortader, iii) PLK. Die Lymphadenektomie der parapylorischen Region (der großen Kurvatur) erfolgte als gesondertes Präparat unter Mitnahme des perigastrischen Fettgewebes. Außer den PLK wurden keine weiteren Lymphknotenstationen gesondert untersucht.

Im Rahmen der pyloruserhaltenden Resektion erfolgte die Durchtrennung des Duodenums immer $2 \mathrm{~cm}$ aboral des Pylorus. Für die Schnellschnittuntersuchung wurde das anhängende parapylorische Fettgewebe der großen Kurvatur auch $2 \mathrm{~cm}$ oral des Pylorus reseziert. Operationstechnisch entspricht dies einem kurzstreckigen Skelettieren der Pylorusregion auf der großen Kurvatur. Bei Tumorbefall der PLK erfolgte eine Nachresektion des distalen Magens im Sinne einer klassischen Whipple-Operation und Rekonstruktion mit Omega-Schlinge und Braunscher Anastomose.

Die Befunde aller Patienten wurden prospektiv in einer Datenbank erfasst. Im Rahmen dieser Studie erfolgte die retrospektive Ergänzung um histologische Detailbefunde. Zur Klassifikation perioperativer Komplikationen wird routinemäßig das Accordion-System eingesetzt [7]. Darüber hinaus wird das Vorliegen einer Pankreasfistel entsprechend der Klassifikation der International Study Group for Pancreatic Fistula (ISGPF) definiert [8]. Es erfolgte eine Gruppierung von Pankreaskarzinomen und extrapankreatischen Karzinomen (distales Gallengangskarzinom, Ampullenkarzinom, Duodenalkarzinom). Zur Datenanalyse wurde das Statistikprogramm IBM SPSS Statistics (Version 19) (IBM Deutschland $\mathrm{GmbH}$, Ehningen, Deutschland) eingesetzt.

\section{Ergebnisse}

In 105 Fällen war eine PPPD indiziert und geplant. Aufgrund der intraoperativen Schnellschnittergebnisse wurde in 8 Fällen mit Pankreaskopfkarzinom der Eingriff geändert. In 4 Fällen mit tumorpositiven PLK erfolgte eine distale Magenresektion. In 4 weiteren Fällen (alle PLK-negativ) mit Tumornachweis am Pankreasabsetzungsrand erfolgte eine Pankreatektomie mit pyloruserhaltender Rekonstruktion.

Die histologische Aufarbeitung zeigte als Primärtumor 72 Pankreaskopfkarzinome und 33 extrapankreatische Karzinome (20 distale Gallengangskarzinome, 8 Ampullenkarzinome, 5 Duodenalkarzinome). Über alle Tumorentitäten hinweg ergab die histologische TNM(tumor-nodemetastasis)-Klassifikation Folgendes: Etwa 90\% aller Patienten hatten lokal fortgeschrittene Tumoren im Stadium T1 ( $\mathrm{n}=4 ; 3,8 \%)$, T2 (n= 8; 7,6\%), T3 $(\mathrm{n}=83 ; 79 \%)$ und T4 $(\mathrm{n}=10 ; 9,5 \%)$. Annähernd $70 \%$ aller Patienten waren nodalpositiv, d.h. N0 $(n=32 ; 30,5 \%)$. Als Bestätigung unserer präoperativen Diagnostik fanden sich in keinem Fall Metastasen, d.h. M0 ( $\mathrm{n}=105 ; 100 \%)$. Das Grading war: G1 ( $\mathrm{n}=2$; $1,9 \%), \mathrm{G} 2(\mathrm{n}=52 ; 49,5 \%), \mathrm{G} 3(\mathrm{n}=51 ; 48,6 \%)$. In annähernd einem Viertel aller Pankreaskopfresektionen wurden histopathologische Tumorresiduen am Resektionsrand beschrieben, d.h. R0 ( $\mathrm{n}=78 ; 74,3 \%)$.

Die Morbidität in der Gesamtgruppe betrug 61,9\%. Hiervon wurden zwei Drittel aller Komplikationen in Gruppe 1 
Tab. 1. Von 105 Patienten waren 73 nodalpositiv, allerdings hatten nur 4 Patienten mit Pankreaskopfkarzinom jeweils einen positiven parapylorischen Lymphknoten (PLK)

\begin{tabular}{lcll}
\hline & Alle Patienten & $\begin{array}{l}\text { Nodalpositive } \\
\text { Patienten }\end{array}$ & $\begin{array}{l}\text { Davon positive } \\
\text { PLK }\end{array}$ \\
\hline Pankreaskarzinom & 72 & 54 & 4 \\
Distales Gallengangskarzinom & 20 & 13 & 0 \\
Ampullenkarzinom & 8 & 3 & 0 \\
Duodenalkarzinom & 5 & 3 & 0 \\
Gesamt & 105 & 73 & 4 \\
\hline
\end{tabular}

Tab. 2. Bei 105 Patienten wurden insgesamt 2391 Lymphknoten (LK) untersucht; hiervon waren 325 parapylorische Lymphknoten (PLK)

\begin{tabular}{|c|c|c|c|c|c|c|}
\hline & \multicolumn{3}{|c|}{ Anzahl aller LK (inkl. parapylorisch) } & \multicolumn{3}{|l|}{ PLK } \\
\hline & gesamt & $\mathrm{N}+$ & Anteil, \% & gesamt & $\mathrm{N}+$ & Anteil, \% \\
\hline Pankreaskarzinom & 1699 & 269 & 16 & 207 & 4 & 2 \\
\hline Distales Gallengangskarzinom & 448 & 65 & 15 & 65 & 0 & 0 \\
\hline Ampullenkarzinom & 142 & 18 & 13 & 25 & 0 & 0 \\
\hline Duodenalkarzinom & 102 & 5 & 5 & 28 & 0 & 0 \\
\hline Gesamt & 2391 & 357 & 15 & 325 & 4 & 1 \\
\hline
\end{tabular}

(mild) und Gruppe 2 (moderat) nach Accordion eingestuft. Die Mortalität betrug 3,8\% ( $\mathrm{n}=4)$. Perioperative Pankreasfisteln (Typ A-C) traten in 21 Fällen (21\%) auf.

Der Anteil nodalpositiver Patienten betrug beim Pankreaskopfkarzinom $\mathrm{n}=54(75 \%)$ und bei den extrapankreatischen Karzinomen n = 19 (58\%). Zusammenfassend waren 73 Patienten nodalpositiv (median 4 LK, min. 1 LK, max. 15 LK). Bezogen auf die Primärtumoren waren positive PLK nur beim Pankreaskopfkarzinom vorhanden $(n=4)$. Bei allen anderen Primärtumoren wurden keine positiven PLK nachgewiesen (nicht signifikant/n.s.). Beim Pankreaskarzinom waren somit 5\% aller Patienten bzw. in der Subgruppe der nodalpositiven Patienten $8 \%$ betroffen (Tab. 1).

Die Gesamtzahl aller untersuchten LK betrug $n=2391$ (median 21, min. 5, max. 57). Der Anteil tumorpositiver LK war $14,9 \%(n=357)$. In der Gruppe der PLK waren lediglich 4 von 325 untersuchten PLK tumorpositiv (1,2\%).

Im parapylorischen Fettgewebe bestand in keinem Fall der palpatorische Verdacht auf eine Tumorinfiltration. In 13 Fällen wurden in der histopathologischen Aufarbeitung keine LK nachgewiesen. In den übrigen 92 Fällen betrug die Anzahl der untersuchten PLK im Median 2 (min. 0, max. 17). In der Gruppe der tumorpositiven PLK ( $n=4$ Patienten) wurde im parapylorischen Gewebepräparat jeweils nur ein tumorbefallener LK gefunden (Tab. 2).

Bei der feingeweblichen Untersuchung der distalen Magenpräparate konnten keine Tumorresiduen nachgewiesen werden. In operationstechnischer Hinsicht ergaben sich keine relevanten Durchblutungsstörungen durch die parapylorische Lymphadenektomie. Somit bestand in keinem Fall die technische Notwendigkeit einer distalen Magenresektion.

\section{Diskussion}

Im hier vorgestellten Konzept erhält die Lymphadenektomie der PLK die Funktion eines «Sentinel». Bei Tumorbefall der PLK erfolgt die distale Magenresektion. Das Vorgehen basiert mehr auf theoretischen Überlegungen und ist bislang kaum in Studien untersucht.

Hinsichtlich des Tumorbefalls von PLK beim Pankreaskarzinom wird je nach Studie eine Rate von 6-20\% beschrieben. Die übrigen perigastrischen Lymphknotenstationen hingegen sind sozusagen nie tumorbefallen [4-6, 9]. Von daher erscheint die Entfernung der PLK beim Pankreaskarzinom onkologisch sinnvoll. In unserer Studie waren $8 \%$ aller nodalpositiven Patienten betroffen, wobei ein histologischer Tumornachweis jeweils lediglich in einem LK erfolgte. Eine makroskopisch tastbare Tumorformation oder Infiltration des distalen Magens bestanden hier nicht.

Lokale Tumorfreiheit ist das grundsätzliche Ziel einer onkologischen Pankreaskopfresektion und stellt die einzige Chance auf Heilung dar. Es bleibt aber ein bekanntes klinisches Problem, dass frühe Rezidive auch nach R0-Resektion auftreten. Die wahrscheinlichste Erklärung sind Tumorresiduen, die der konventionellen histopathologischen Untersuchung entgehen. Bekannt sind lymphatische Mikrometastasen, die bei differenzierter histologischer Spezialuntersuchung in bis zu 78\% der vermeintlich tumorfreien LK gefunden werden können [10]. Ein weiteres Problem sind randbildende Tumormanifestationen der retroperitonealen Schnittfläche. Standardisierte Untersuchungen der Resektionsflächen konnten hier Tumormanifestationen in bis zu 76\% der Resektionspräparate nachweisen [11, 12]. Insgesamt ist der Anteil der tatsächlichen R0-Patienten vermutlich viel geringer als bislang angenommen. Okkulte Tumorzellen können multipel lokalisiert sein und ein Rezidiv verursachen. 
In den distalen Magenresektaten unserer Studie ergab sich in keinem Fall der Anhalt für eine Tumorinfiltration. Ein individueller onkologischer Nutzen für unsere Patienten ist nicht belegbar. In allen großen Studien hat sich kein Überlebensvorteil durch eine distale Magenresektion im Sinne einer Whipple-Operation gezeigt $[1,2,13]$. Die einfachere und funktionell bessere PPPD ist daher zum Standardverfahren geworden. In neueren Arbeiten wird vor dem Hintergrund einer verbesserten früh postoperativen Magenentleerung der Pyloruserhalt erneut diskutiert. Das als SSPPD (subtotal stomachpreserving pancreatoduodenectomy) beschriebene Verfahren soll im früh postoperativen Verlauf Vorteile bieten [14, 15]. Bei dieser Technik wird lediglich der Pylorusring reseziert und der Magen fast vollständig erhalten. Die parapylorische Lymphadenektomie würde sich damit von selbst ergeben.

Nach unserer Beobachtung hat die parapylorische Lymphadenektomie keinen relevanten Einfluss auf die Durchblutung der Pylorusregion. Kontraindikationen hierzu ergeben sich somit nicht, während der operativ technische Aufwand gering ist. Die parapylorische Lymphadenektomie kann daher unabhängig vom Pyloruserhalt bzw. vom Ausmaß der distalen Magenresektion durchgeführt werden. Sie kann in Einzelfällen beim Pankreaskarzinom eine verbesserte Tumorfreiheit bewirken.

\section{Schlussfolgerung}

Die eigenen Daten bestätigen die Ergebnisse aus der Literatur, wonach beim Pankreaskarzinom ein geringer Anteil an Patienten einen Tumorbefall der PLK hat. Die Resektion erscheint somit sinnvoll. Der Nutzen einer intraoperativen Schnellschnittuntersuchung mit nachfolgender Konsequenz für eine etwaige distale Magenresektion ist anhand unserer Daten nicht belegbar.

\section{Disclosure Statement}

Keine Interessenkonflikte.

\section{Literatur}

$D_{1}$ Tran KT, Smeenk HG, van Eijck CH, Kazemier G, Hop WC, Greve JW, Terpstra OT, Zijlstra JA, Klinkert P, Jeekel H: Pylorus preserving pancreaticoduodenectomy versus standard Whipple procedure: a prospective, randomized, multicenter analysis of 170 patients with pancreatic and periampullary tumors. Ann Surg 2004;240:738-745.

- 2 Riall TS, Cameron JL, Lillemoe KD, Campbell KA, Sauter PK, Coleman J, Abrams RA, Laheru D, Hruban RH, Yeo CJ: Pancreaticoduodenectomy with or without distal gastrectomy and extended retroperitoneal lymphadenectomy for periampullary adenocarcinoma - part 3: update on 5-year survival. J Gastrointest Surg 2005;9:1191-1204.

$\checkmark 3$ Diener MK, Knaebel HP, Heukaufer C, Antes G, Buchler MW, Seiler CM: A systematic review and meta-analysis of pylorus-preserving versus classical pancreaticoduodenectomy for surgical treatment of periampullary and pancreatic carcinoma. Ann Surg 2007;245:187-200.

4 Heise JW, Becker H, Borchard F, Roher HD: Zum Radikalitätsrisiko der pyloruserhaltenden Duodenopankreatektomie beim duktalen Karzinom. Chirurg 1994;65:780-784
5 Mu DQ, Peng YS, Wang FG, Xu QJ: Significance of perigastric lymph node involvement in periampullary malignant tumor. World J Gastroenterol 2004:10:614-616.

6 Hopt UT: Therapie des Pankreaskopfkarzinoms Zentralbl Chir 2006;131:115-120.

7 Strasberg SM, Linehan DC, Hawkins WG: The accordion severity grading system of surgical complications. Ann Surg 2009;250:177-186.

$\checkmark 8$ Bassi C, Falconi M, Molinari E, Mantovani W, Butturini G, Gumbs AA, Salvia R, Pederzoli P: Ductto-mucosa versus end-to-side pancreaticojejunostomy reconstruction after pancreaticoduodenectomy: results of a prospective randomized trial. Surgery 2003;134:766-771.

$\checkmark 9$ Gerdes B, Ramaswamy A, Bartsch DK, Rothmund M: Peripyloric lymph node metastasis is a rare condition in carcinoma of the pancreatic head. Pancreas 2005;31:88-92.

10 Kayahara M, Funaki K, Tajima H, Takamura H, Ninomiya I, Kitagawa H, Ohta T: Surgical implication of micrometastasis for pancreatic cancer. Pancreas 2010;39:884-888.
1 Esposito I, Kleeff J, Bergmann F, Reiser C, Herpel E, Friess H, Schirmacher P, Buchler MW: Most pancreatic cancer resections are R1 resections. Ann Surg Oncol 2008;15:1651-1660.

12 Rau BM, Moritz K, Schuschan S, Alsfasser G, Prall $\mathrm{F}$, Klar E: R1 resection in pancreatic cancer has significant impact on long-term outcome in standardized pathology modified for routine use. Surgery 2012;152:S103-S111.

13 Iqbal N, Lovegrove RE, Tilney HS, Abraham AT, Bhattacharya S, Tekkis PP, Kocher HM: A comparison of pancreaticoduodenectomy with pylorus preserving pancreaticoduodenectomy: a meta-analysis of 2822 patients. Eur J Surg Oncol 2008;34: 1237-1245.

14 Hayashibe A, Kameyama M, Shinbo M, Makimoto $\mathrm{S}$ : The surgical procedure and clinical results of subtotal stomach preserving pancreaticoduodenectomy (SSPPD) in comparison with pylorus preserving pancreaticoduodenectomy (PPPD). J Surg Oncol 2007;95:106-109.

15 Kurahara H, Takao S, Shinchi H, Mataki Y, Maemura K, Sakoda M, Ueno S, Natsugoe S: Subtotal stomach-preserving pancreaticoduodenectomy (SSPPD) prevents postoperative delayed gastric emptying. J Surg Oncol 2010;102:615-619. 